\title{
Influencia de las Características Sociodemográficas y Económicas en el Aborto de la Adolescente Atendida en el Hospital Hipólito Unanue de Tacna-2008
}

RESPONSABLE: Mgr. Eloina Tejada Monroy

RESUMEN. El presente trabajo de investigación se realizó con el objetivo de determinar si las características sociodemográficas y económicas influyen en el aborto de la adolescente atendida en el Hospital Hipólito Unanue. La ciudad de Tacna, presenta un gran porcentaje de padres de familia que se dedican al comercio, y esta actividad económica que desempeñan les demanda bastante tiempo, hecho que no les permite orientar de manera adecuada y oportuna a sus hijos adolescentes, los que desarrollan prácticas y conductas dañinas, como el alcoholismo, la drogadicción y principalmente el inicio de prácticas coitales a temprana edad, sin conocimientos ni la responsabilidad del caso. Por lo tanto, estos hechos traen como consecuencia los embarazos no deseados en los adolescentes, los mismos que recurren al aborto como solución.

Después de haber realizado la aplicación de la encuesta, se concluyó que las características sociodemográficas y económicas que más influyen en el aborto adolescente son: edad entre $15-19$ años $(100 \%)$, procedencia de zona urbana $(78,95 \%)$, grado de instrucción secundaria completa (47,37\%), ocupación estudiante $(36,84 \%)$; religión católica (73,68\%), convivencia con su familia $(52,63 \%)$, ingreso económico medio (42,11\%). Así mismo se ha encontrado que la principal causa de aborto es el rechazo familiary el rechazo social.
MIEMBRo: $M g r$ Ingrid Maria Manrique Tejada

ABSTRACT The present work of investigation, realized with the aim to determine if the characteristics sociodemográficas and economic influence in the abortion of the teenager attended in the Hospital Hipólito Unánue. The city of Tacna, presents a big percentage of parents of family, that devote to the trade, And this economic activity that exert sues them quite a lot of time, fact that does not allow them orientar of felicitous and timely way to his children teenagers, those that develop practical and harmful behaviours, as the alcoholism, the drogadicción and mainly the start of practices coitales to early age, without knowledges and this economic activity that exert sues them quite a lot of time, fact that does not allow them orientar neither the responsibility of the case. Therefore, these facts carry like consequence the no wished pregnancies in the teenagers, the same that resort to the abortion like solution

After having realized the application of the survey, concluded that the characteristic partnerdemographical and economic that more influence in the abortion teenager are: age between 15-19 years $(100 \%)$, procedencia of urban zone $(78,95 \%)$, degree of complete secondary instruction (47,37\%), occupation student $(36,84 \%)$, Catholic religion (73,68\%), convivencia with his family $(52,63 \%)$, half economic entry $(42,11 \%)$. Likewise has found that the main cause of abortion is the refuse familiar and the refuse social.
INTRODUCCIÓN. Siendo el aborto una de las primeras causas de morbimortalidad de este grupo etario y el incremento de fecundidad en las adolescentes y al gran riesgo que este grupo se expone. Frente a esta problemática interesa investigar las características del porqué se produce este fenómeno y si estas tienen relación directa con las condiciones socioeconómicas y geográficas ,y así crear pautas y aplicarlas con el fin de mejorar la atención integral de la salud reproductiva y lograr que se interrelacione con otros factores sociales y así nos permitirá conocer las principales características sociodemográficas y económicas de los servicios de la salud sexual y reproductiva que influyen con mayor frecuencia en el aborto de este grupo etario tan vulnerable como es el de las adolescentes.

\section{MARCOTEÓRICO}

\section{Adolescencia}

La adolescencia es el período crucial del desarrollo del ser humano que incluye cambios físicos y psicológicos que determinan la transformación del niño a adulto. Según la OMS, la adolescencia comprende el grupo etario de 10 a 19 años. La OMS clasifica la adolescencia como: adolescencia temprana: de los 10-14 años y adolescencia tardía: de los 15-19 años.

Embarazo en la adolescencia

El embarazo en la adolescencia se define como aquella gestación que ocurre durante los 2 primeros años ginecológicos de la mujer (edad ginecológica $0=$ edad de la menarquia) y/o cuando la adolescente mantiene la completa dependencia social y económica de la familia parental. Ha sido llamado también el síndrome del fracaso o la puerta de entrada al ciclo de la pobreza. (OPS. Manual de medicina de la adolescencia. 1992).

Aborto

Etimológicamente deriva de la composición de un prefijo y una raiz latina, $\mathrm{AB}$ : privación y ORTUS: nacimiento: ABORTUS, palabra compuesta que traduciría $\sin$ nacimiento. Se denomina aborto a la 
interrupción espontánea o provocada del embarazo antes de la vigésima semana de gestación con un peso del producto inferior a 500 gramos y se llama producto del aborto.

\section{OBJETIVOS}

\section{Objetivo General}

Determinar las características sociodemográficas y económicos que influyen en el aborto de la adolescente atendida en el Hospital Hipólito Unanue de Tacna, en el tercer trimestre de 2008.

\section{Objetivos Específicos}

1. Determinar el número de adolescentes que son atendidas por aborto en el Hospital Hipólito Unanue en el tercer trimestre 2008

2. Identificar las condiciones sociodemográficas y económicas de las adolescentes atendidas por aborto en el Hospital Hipólito Unánue de Tacna, en el tercer trimestre de 2008

\section{MATERIALES Y MÉTODOS}

Tipo de investigación

Básica.

\section{Diseño de la investigación}

Es investigación descriptiva.

\section{Población y muestra de estudio}

La muestra estuvo constituida por todas las adolescentes entre 10 a 19 años que fueron atendidas en el servicio gineco-obstetricia del Hospital Hipólito Unanue siendo un total de 19 .

\section{RESULTADOS}

Cuadro $\mathrm{N}^{\circ} 01$ Edad de Adolescentes Atendidas por Aborto en Relación con la Causa de Aborto. Hospital Hipólito Unamue de Tacna. Tercer Trimestre de 2008

\begin{tabular}{|c|c|c|c|c|c|c|}
\hline \multirow{3}{*}{$\begin{array}{c}\text { CAUSA DE } \\
\text { ABORTO }\end{array}$} & \multicolumn{4}{|c|}{ EDAD } & \multirow{2}{*}{\multicolumn{2}{|c|}{ TOTAL }} \\
\hline & \multicolumn{2}{|c|}{$10-14$ años } & \multicolumn{2}{|c|}{$15-19$ años } & & \\
\hline & $\mathrm{N}^{0}$ & $\%$ & $\mathrm{~N}^{\circ}$ & $\%$ & $\mathrm{~N}^{0}$ & $\%$ \\
\hline $\begin{array}{c}\text { Embarazo no } \\
\text { planificado }\end{array}$ & 0 & 0 & 2 & 10,53 & 2 & 10,53 \\
\hline Rochazo Familiar & 0 & 0 & 7 & 36,84 & 7 & 36,84 \\
\hline Rechaze Social & 0 & 0 & 6 & 31.58 & 6 & 31,58 \\
\hline Violación & 0 & 0 & 4 & 21,05 & 4 & 21,05 \\
\hline TOTAL & 0 & 0 & 19 & 100,00 & 19 & 100,00 \\
\hline
\end{tabular}

Fuente: Encuesta aplicada.

\section{Interpretación}

El Cuadro $N^{\circ} 01$ muestra que de todas las adolescentes entrevistadas (100\%), se encuentran entre las edades de 15 - 19 años, de las cuales el $36,84 \%$ abortaron por rechazo familiar seguido de un $31,58 \%$ por rechazo social.
Gráfico $\mathrm{N}^{\circ} 01$ Edad de Adolescentes Atendidas por Aborto en Relación con la Causa de Aborto. Hospital Hipólito Unánue de Tacna, Tercer Trimestre de 2008

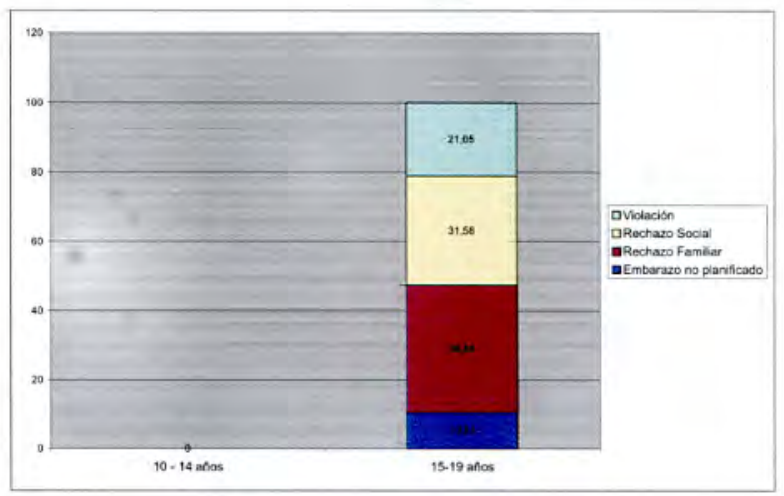

Cuadro $\mathrm{N}^{\circ} 02$ Procedencia de Adolescentes Atendidas por Aborto en Relación con la Causa de Aborto. Hospital Hipólito Unanue de Tacna, Tercer Trimestre de 2008.

\begin{tabular}{|c|c|c|c|c|c|c|}
\hline \multirow{3}{*}{$\begin{array}{l}\text { CAUSA DE } \\
\text { ABORTO }\end{array}$} & \multicolumn{4}{|c|}{ PROCEDENCIA } & \multirow{2}{*}{\multicolumn{2}{|c|}{ TOTAL }} \\
\hline & \multicolumn{2}{|c|}{ URBANO } & \multicolumn{2}{|c|}{ RURAL } & & \\
\hline & $\mathrm{N}^{\circ}$ & $\%$ & $\mathrm{~N}^{0}$ & $\%$ & $\mathrm{~N}^{0}$ & $\%$ \\
\hline $\begin{array}{l}\text { Embarazo no } \\
\text { planificado }\end{array}$ & 2 & 10,53 & 0 & 0,00 & 2 & 10,53 \\
\hline Rechazo Familiar & 5 & 26,32 & 2 & 10,53 & 7 & 36,84 \\
\hline Rechazo Social & 6 & 31,58 & 0 & 0,00 & 6 & 31,58 \\
\hline Violación & 2 & 10,53 & 2 & 10,53 & 4 & 21,05 \\
\hline TOTAL & 15 & 78,95 & 4 & 21,05 & 19 & 100,00 \\
\hline
\end{tabular}

Fuente: Elaboración propia.

\section{Interpretación}

El Cuadro $\mathrm{N}^{\circ} 02$ muestra que de todas las adolescentes entrevistadas, el 78,95\% proceden de área urbana y el $21,05 \%$ proceden de área rural. Del total de las adolescentes que proceden de área urbana se observa que la mayoria $(31,58 \%)$ abortaron por rechazo social.

Gráfico $\mathrm{N}^{\circ} 02$ Procedencia de Adolescentes Atendidas por Aborto en Relación con la Causa de Aborto. Hospital Hipólito Unanue de Tacna, Tercer Trimestre de 2008

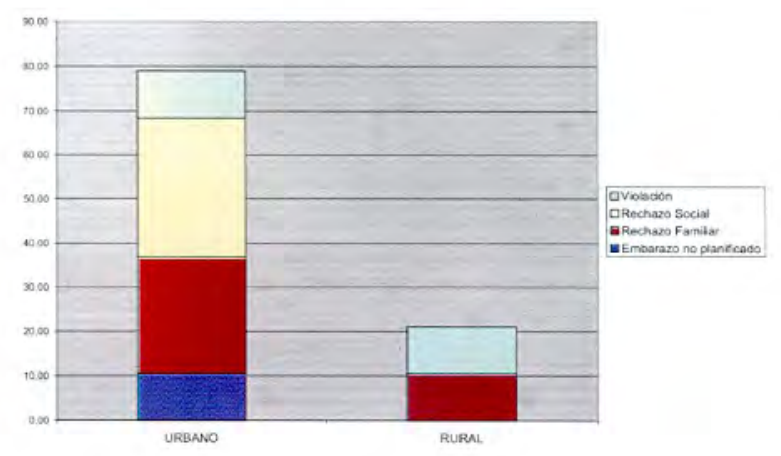


Cuadro $\mathrm{N}^{\circ} 03$ Ingreso Económico de Adolescentes Atendidas por Aborto en Relación con la Causa de Aborto. Hospital Hipólito Unanue de Tacna, Tercer Trimestre de 2008.

\begin{tabular}{|c|c|c|c|c|c|c|c|c|}
\hline \multirow{2}{*}{$\begin{array}{c}\text { CAUSA DE } \\
\text { ABORTO }\end{array}$} & \multicolumn{5}{|c|}{ ALTO } & \multicolumn{2}{|c|}{ MEDIO } & \multicolumn{2}{c|}{ BAJO } & \multicolumn{2}{c|}{ TOTAL } \\
\cline { 2 - 8 } & $\mathrm{N}^{\circ}$ & $\%$ & $\mathrm{~N}^{\circ}$ & $\%$ & $\mathrm{~N}^{\circ}$ & $\%$ & $\mathrm{~N}^{\circ}$ & $\%$ \\
\hline $\begin{array}{c}\text { Embarazo no } \\
\text { planificado }\end{array}$ & 0 & 0,00 & 1 & 5,26 & 1 & 5,26 & 2 & 10,53 \\
\hline $\begin{array}{c}\text { Rechazo } \\
\text { Familiar }\end{array}$ & 3 & 15,79 & 3 & 15,79 & 1 & 5,26 & 7 & 36,84 \\
\hline $\begin{array}{c}\text { Rechazo } \\
\text { Social }\end{array}$ & 3 & 15,79 & 2 & 10,53 & 1 & 5,26 & 6 & 31,58 \\
\hline $\begin{array}{c}\text { Violación } \\
\text { NOTAL }\end{array}$ & 1 & 5,26 & 2 & 10,53 & 1 & 5,26 & 4 & 21,05 \\
\hline TOTAL & 76,84 & 8 & 42,11 & 4 & 21,05 & 19 & 100,00 \\
\hline
\end{tabular}

Fuente: Encuesta Aplicada.

\section{Interpretación}

El Cuadro $\mathrm{N}^{\circ} 03$ muestra que de todas las adolescentes entrevistadas, el $42,11 \%$ tienen un ingreso económico medio, seguido de un $36,84 \%$ con un ingreso económico alto. Así mismo se observa que la principal causa de aborto es por rechazo familiar.

Gráfico $\mathrm{N}^{\circ} 3$ Ingreso Económico de Adolescentes Atendidas por Aborto en Relación con la Causa de Aborto. Hospital Hipólito Unanue de Tacna, Tercer Trimestre de 2008.

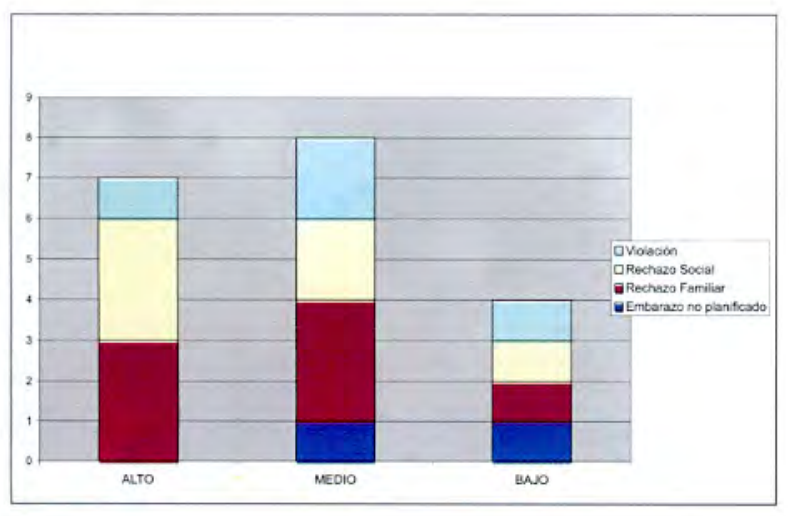

Cuadro $\mathrm{N}^{\circ} 04$ Grado de Instruccción de Adolescentes Atendidas por Aborto en Relación a La Causa de Aborto. Hospital Hipólito Unanue de Tacna. Tercer Trimestre 2008

\begin{tabular}{|c|c|c|c|c|c|c|c|c|c|c|c|c|}
\hline \multirow{3}{*}{$\begin{array}{l}\text { CAUSA DE } \\
\text { ABORTO }\end{array}$} & \multicolumn{10}{|c|}{ GRADO DE INSTRLCCIÓN } & \multirow{2}{*}{\multicolumn{2}{|c|}{ TOTAL }} \\
\hline & \multicolumn{2}{|c|}{ ANALFABETA } & \multicolumn{2}{|c|}{$\begin{array}{l}\text { PRIMARIA } \\
\text { INOOMPLETA }\end{array}$} & \multicolumn{2}{|c|}{$\begin{array}{l}\text { PRIMARIA } \\
\text { COMPLETA }\end{array}$} & \multicolumn{2}{|c|}{$\begin{array}{l}\text { SECUNDRIA } \\
\text { COMPLETA }\end{array}$} & \multicolumn{2}{|c|}{ SUPERIOR } & & \\
\hline & $\mathrm{N}^{\mathrm{N}}$ & $\%$ & $\mathrm{~N}^{\mathrm{N}}$ & $\%$ & $\mathrm{~N}^{\circ}$ & $\%$ & $N^{\circ}$ & $\%$ & $N^{\circ}$ & $\%$ & $N^{\circ}$ & $\%$ \\
\hline $\begin{array}{l}\text { Embarazo no } \\
\text { planificado }\end{array}$ & 0 & 0,00 & 0 & 0,00 & 0 & 0,00 & 0 & 0,00 & 2 & 10,53 & 2 & 10,53 \\
\hline $\begin{array}{l}\text { Rechazo } \\
\text { Farniliar }\end{array}$ & 0 & 0,00 & 0 & 0,00 & 0 & 0,00 & 4 & 21,05 & 3 & 15,79 & 7 & 36,84 \\
\hline $\begin{array}{l}\text { Rechuzo } \\
\text { Social }\end{array}$ & 0 & 0,00 & 0 & 0,00 & 1 & 5,26 & 3 & 15,79 & 2 & 10,53 & 6 & 31,58 \\
\hline Violación & 0 & 0,00 & 1 & 5,26 & 0 & 0,00 & 2 & 10,53 & 1 & 5,26 & 4 & 21,05 \\
\hline TOTAL & 0 & 0,00 & 1 & 5,26 & 1 & 5,26 & 9 & 47,37 & 8 & 42,11 & 19 & 100,00 \\
\hline
\end{tabular}

\section{Interpretación}

El Cuadro $\mathrm{N}^{\circ} 04$ muestra que de todas las adolescentes entrevistadas, el $47.37 \%$ tienen grado de instrucción secundaria completa, seguido de un $42.11 \%$ con educación superior y con un menor porcentaje (1\%) adolescentes con primaria incompleta y completa. Asi mismo tenemos que de las adolescentes con grado de instrucción secundaria completa abortaron por rechazo familiar $(21.05 \%)$.

Gráfico $\mathrm{N}^{\circ} 04$ Grado de Instrucción de Adolescentes Atendidas por Aborto en Relación a la Causa de Aborto. Hospital Hipólito Unanue de Tacna. Tercer Trimestre 2008

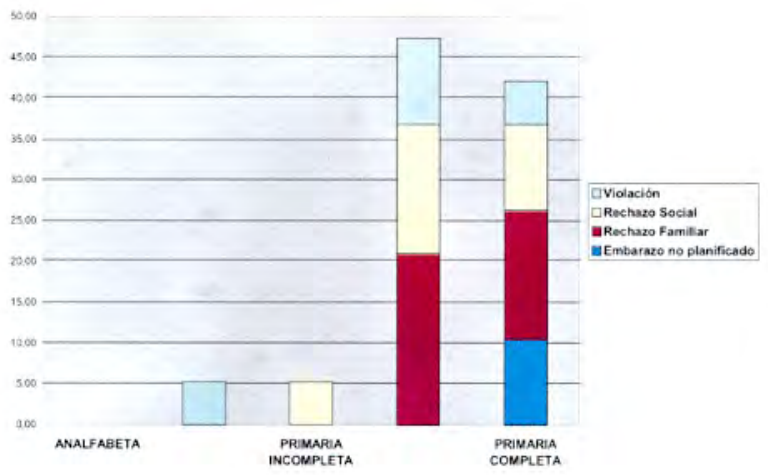

Cuadro $\mathrm{N}^{\circ} 05$ Ocupación de Adolescentes Atendidas por Aborto en Relación a la Causa de Aborto. Hospital Hipólito Unanue de Tacna. Tercer Trimestre 2008

\begin{tabular}{|c|c|c|c|c|c|c|c|c|c|c|c|c|}
\hline \multirow{3}{*}{$\begin{array}{c}\text { CALSA } \\
\text { DE } \\
\text { ABORTO }\end{array}$} & \multicolumn{10}{|c|}{ OCUPACIÓN } & \multirow{2}{*}{\multicolumn{2}{|c|}{ TOTAL }} \\
\hline & \multicolumn{2}{|c|}{ ESTUDIANIE } & \multicolumn{2}{|c|}{ COMERCIANTES } & \multicolumn{2}{|c|}{ DEPENDIENTES } & \multicolumn{2}{|c|}{\begin{tabular}{|c|} 
EMPLEADA \\
DEL \\
HOGAR
\end{tabular}} & \multicolumn{2}{|c|}{ NINGUNA } & & \\
\hline & $\mathrm{N}^{\circ}$ & $\%$ & $N^{p}$ & $\%$ & $\mathrm{~N}^{\circ}$ & $\%$ & $N^{n}$ & $\%$ & $N^{\circ}$ & $\%$ & $\mathrm{~N}^{\mathrm{v}}$ & $\%$ \\
\hline $\begin{array}{c}\text { Embaraco } \\
\text { no } \\
\text { planificado }\end{array}$ & 0 & 0,00 & 0 & 0,00 & 0 & 0,00 & 1 & 5,26 & 1 & 5,26 & 2 & 10.53 \\
\hline $\begin{array}{l}\text { Rechazo } \\
\text { Familiar }\end{array}$ & 4 & 21,05 & 0 & $0, \infty 0$ & 0 & 0,00 & 1 & 5,26 & 2 & 10,53 & 7 & 36,84 \\
\hline $\begin{array}{l}\text { Rechizoo } \\
\text { Social }\end{array}$ & 3 & 15,79 & 1 & 5,26 & 1 & 5,26 & 0 & 0,00 & 1 & 5.26 & 6 & 31,58 \\
\hline Violación & 0 & 0,00 & 2 & 10,53 & 0 & 0,00 & 2 & 10,53 & 0 & 0,00 & 4 & 21,05 \\
\hline TOTAL & 7 & 36,84 & 3 & 15,79 & 1 & 5,26 & 4 & 21.05 & 4 & 21,05 & 19 & 100,00 \\
\hline
\end{tabular}

Fuente: Encuesta Aplicada

\section{Interpretación}

El Cuadro $\mathrm{N}^{\circ} 05$ muestra que de todas las adolescentes entrevistadas, el $36.84 \%$ son estudiantes, seguido de un $21.05 \%$ empleadas del hogar. Así mismo se observa que la principal causa de aborto corresponde a rechazo familiar $(21.05 \%)$. 
Gráfico $\mathrm{N}^{\circ} 05$ Ocupación de Adolescentes Atendidas por Aborto en Relación a la Causa de Aborto. Hospital Hipólito Unanue de Tacna. Tercer Trimestre 2008

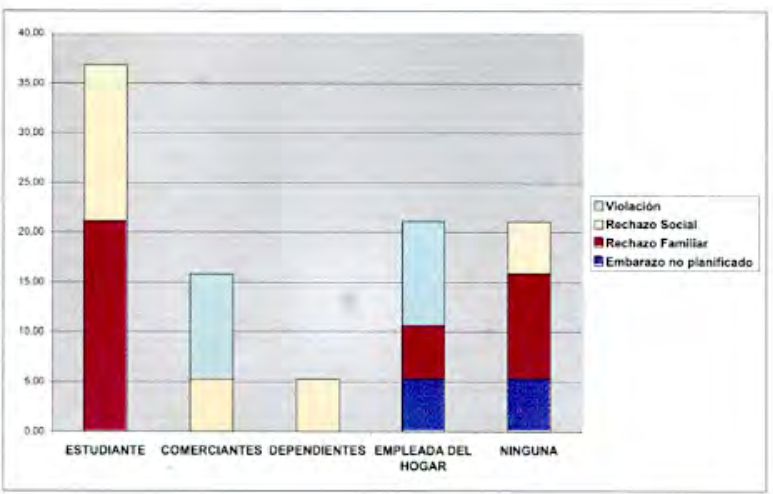

Cuadro $\mathrm{N}^{\circ} 06$ Religión de Adolescentes Atendidas por Aborto en Relación a la Causa de Aborto. Hospital Hipólito Unanue de Tacna. Tercer Trimestre 2008

\begin{tabular}{|c|c|c|c|c|c|c|c|c|c|c|}
\hline \multirow{3}{*}{$\begin{array}{l}\text { CAUSA DE } \\
\text { ABORTO }\end{array}$} & \multicolumn{8}{|c|}{ RELIGIÓN } & \multirow{2}{*}{\multicolumn{2}{|c|}{ TOTAL }} \\
\hline & \multicolumn{2}{|c|}{ CATÓlica } & \multicolumn{2}{|c|}{ EVANGÉLICA } & \multicolumn{2}{|c|}{$\begin{array}{l}\text { TESTIGO DE } \\
\text { JEHOVA }\end{array}$} & \multicolumn{2}{|c|}{ OTRAS } & & \\
\hline & $\mathrm{N}^{2}$ & $\%$ & $\mathrm{~N}^{*}$ & $\%$ & $\mathrm{~N}^{\circ}$ & $\%$ & $\mathrm{~N}^{*}$ & $\%$ & $\mathrm{~N}^{\circ}$ & $\%$ \\
\hline $\begin{array}{l}\text { Embarazo no } \\
\text { platrificado }\end{array}$ & 2 & 10,53 & 0 & 0,00 & 0 & 0.00 & 0 & 0,00 & 2 & 10.53 \\
\hline $\begin{array}{l}\text { Rechazo } \\
\text { Familiar }\end{array}$ & 4 & 21,05 & 2 & 10,53 & 0 & 0.00 & 1 & 5.26 & 7 & 36,84 \\
\hline Rechazo Social & 5 & 26,32 & 0 & 0,00 & 1 & 5,26 & 0 & 0,00 & 6 & 31,58 \\
\hline Violación & 3 & 15,79 & 1 & 5,26 & 0 & 0,00 & 0 & 0,00 & 4 & 21,05 \\
\hline TOTAL. & 14 & 73,68 & 3 & 15,79 & 1 & 5,26 & 1 & 5,26 & 19 & 100,00 \\
\hline
\end{tabular}

Fuente: Encuesta Aplicada

\section{Interpretación}

El Cuadro $\mathrm{N}^{\circ} 06$ muestra que de todas las adolescentes entrevistadas, el $73.68 \%$ pertenecen a la religión Católica, seguido de un $15.79 \%$ de religión Evangélica. Así mismo se observa que la principal causa de aborto es por rechazo social.
Gráfico No 06 Religión de Adolescentes Atendidas por Aborto en Relación a la Causa de Aborto. Hospital Hipólito Unánue de Tacna. Tercer Trimestre 2008

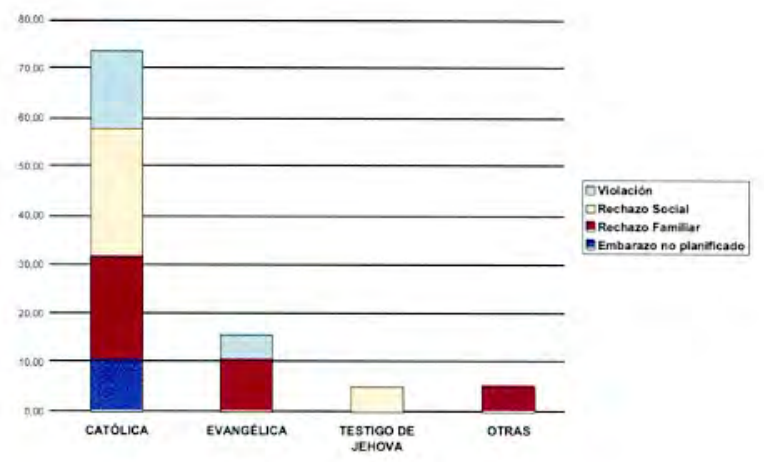

\section{CONCLUSIONES}

Las características sociodemográficas y económicas que más influyen en el aborto adolescente son: edad entre 15 -19 años (100\%), procedencia de zona urbana $(78,95 \%)$, grado de instrucción secundaria completa $(47,37 \%)$, ocupación estudiante $(36,84 \%)$, religión católica $(73,68 \%)$, convivencia con su familia $(52,63 \%)$, ingreso económico medio $(42,11 \%)$ siendo la principal causa del aborto el rechazo familiar y social.

\section{REFERENCIAS BIBLIOGRÁFICAS}

Fernando, D. El aborto Clandestino en el Perú. Hechos y Cifras. Lima. Centro de la Mujer Peruana Flora Tristan y Pathfinder Internacional, 2002.

Instituto Nacional de Estadística e Informática. Encuesta demográfica y de Salud Familiar, ENDES 2000. Lima, INEI, 2001.

López, J; Lugones, M, Valdespino, L. "Aspectos Psicosociales del Embarazo en la Adolescencia". Rev. Cubana Med Gen Integ, 2005.

Flores, C. E. "Factores Socioeconómicos y Contextuales que Determinan la Actividad Reproductiva de las Adolescentes de Colombia". Rev. Panam. Salud Pública, 2005. 